\title{
CHIASSON, Père Anselme, capucin, de la Société Historique Acadienne, Chéticamp - Histoire et Traditions acadiennes. Éditions des Aboiteaux, Moncton, 1961. Préface de Luc Lacourcière. Introduction, Appendices A-B-C-D-E-F, Bibliographie, Table alphabétique, Table des matières, Illustrations, $317 \mathrm{p}$.
}

\section{Lionel Groulx}

Volume 15, numéro 2, septembre 1961

URI : https://id.erudit.org/iderudit/302122ar

DOI : https://doi.org/10.7202/302122ar

Aller au sommaire du numéro

Éditeur(s)

Institut d'histoire de l'Amérique française

ISSN

0035-2357 (imprimé)

1492-1383 (numérique)

Découvrir la revue

Citer ce compte rendu

Groulx, L. (1961). Compte rendu de [CHIASSON, Père Anselme, capucin, de la Société Historique Acadienne, Chéticamp - Histoire et Traditions acadiennes. Éditions des Aboiteaux, Moncton, 1961. Préface de Luc Lacourcière. Introduction, Appendices A-B-C-D-E-F, Bibliographie, Table alphabétique, Table des matières, Illustrations, 317 p.] Revue d'histoire de l'Amérique française, 15(2), 302-304. https://doi.org/10.7202/302122ar d'utilisation que vous pouvez consulter en ligne. 
Chiasson, Père Anselme, capucin, de la Société Historique Acadienne, Chéticamp - Histoire et Traditions acadiennes. Editions des Aboiteaux, Moncton, 1961. Préface de Luc Lacourcière. Introduction, Appendices A-B-C-D-E-F, Bibliographie, Table alphabétique, Table des matières, Illustrations, 317 pages.

Une histoire de paroisse qu'on lira avec pleine satisfaction. Elle est bien faite. Et l'auteur, fils de ce coin de pays, peut en parler en connaisseur; et il a bénéficié d'un sujet de vigoureuse et attachante originalité. Chéticamp, en l'île du Cap-Breton, presque à l'autre bout du Canada, aux rives du Golfe SaintLaurent et de l'Atlantique, ne dirait-on pas une sentinelle française hissée près des falaises du Cabot Trail ? Et comment n'y pas voir une image saisissante de la renaissance d'un peuple? Chéticamp (d'un mot micmac qui veut dire: « rarement plein ») n'existait guère avant la Déportation de 1755 . Ne fréquentent la région que des Indiens micmacs, des pêcheurs de morue bretons et basques, et plus tard, des Jersiais. Des Acadiens errants y arrivent vers 1785-1786, la plupart victimes de la deuxième déportation de 1758, expulsés de l'Ile Saint-Jean et du Cap-Breton. Ils viennent de la Baie-des-Chaleurs, des Iles de la Madeleine, de Saint-Pierre-et-Miquelon. On recense même parmi eux, des Acadiens déportés en France. Que cherchent ces vagabonds ? Un coin retiré où échapper aux exactions, à la rapacité des conquérants. Chéticamp, à l'extrémité nord-ouest du Cap-Breton, se prête on ne peut mieux à ce dessein. Sur ces rivages battus par les vagues du golfe et de l'océan, pas d'autres liens avec le reste du monde, que par le passage du rare missionnaire et l'accostage des goélettes jersiaises. Pour plus de précautions, les réfugiés s'établissent loin des bords de la mer, en des vallées peu commodes, moins accessibles aux chasseurs d'hommes. On se partage le terrain à l'amiable. En 1790 seulement sera-t-il loisible d'obtenir du gouverneur de Sydney des titres officiels de propriété.

De ce petit noyau d'hommes, abreuvés de misère, venus de tous les horizons, qui entreprennent de reconstruire une parcelle de l'Acadie française, le Père Anselme Chiasson nous raconte l'histoire. Histoire pénible en son commencement: vie de petits agriculteurs, vie de pêcheurs et de chasseurs, vie de mineurs, vie de chantiers, vie d'éleveurs de visons. Mais la longue misère a rendu ces hommes débrouillards; ils se donnent peu à peu les artisans, les professionnels dont ils ont besoin; des institutions essentielles naissent, se développent: paroisse, hôpital, école. Il fut un temps, avant 1812, où un seul d'entre eux, ou peu s'en 
faut, savait signer son nom. Leur pire souffrance sera le manque de prêtres, de missionnaires, manque qui se fait sentir au Canada même. Pendant longtemps, pour suppléer à l'absence du prêtre, l'évêque de Québec confère des pouvoirs spéciaux à quelque bon laïc Acadien. L'office du délégué consistera à présider les « messes blanches », à faire chanter, le dimanche, à la chapelle ou à l'église, quelques parties de l'office, à faire réciter le chapelet, à faire chanter des cantiques dont quelques-uns étaient de véritables leçons de catéchisme. Pourtant Mgr Plessis passe, en 1812, en visite pastorale, chez ces isolés qui n'ont jamais vu d'évêque, et ne savent comment manifester leur joie. Peu à peu les missionnaires résidents leur arrivent. Et il en vient de fort remarquables: Augustin-Magloire Blanchet, futur évêque de Walla-Walla en Colombie, l'abbé Antoine Manseau, qui devint grand-vicaire de Montréal, l'abbé René Gaulin, coadjuteur, en 1833, de l'évêque de Kingston. Par où l'on voit que l'évêque de Québec dépêchait aux Acadiens quelques-uns de ses meilleurs sujets. Un chapitre particulièrement intéressant du monographiste est celui où il nous décrit les mœurs de ses co-paroissiens de Chéticamp. A la première époque ces réchappés de l'exil font voir un sens religieux si profond qu'il en devient émouvant. L'absence du prêtre, l'ignorance religieuse, la désolation de leur sort, n'ont rien pu contre leur foi. "On n'est pas martyr pour rien », observe le Père Chiasson. Ces Acadiens ont aussi gardé quelque chose de la vie patriarcale. Les épreuves communes, l'isolement à la pointe extrême du pays, les liens du sang entre ces gens forcément mariés dans la proche parenté, ont longtemps entretenu chez eux un vivant esprit de famille. N'allons pas croire pour autant à une idylle pastorale. Le blasphème n'existe pas chez eux. Mgr Plessis admire leur belle simplicité, leur foi. Et, pour un peu, les comparerait aux chrétiens de la primitive Eglise. Pourtant quelques excès se sont introduits en cette population trop longtemps abandonnée. Les missionnaires dénoncent l'ivrognerie; l'on est trop proche de Saint-Pierre-et-Miquelon, l'on a voyagé aux Antilles; au surplus les magasins des jersiais regorgent d'alcool. L'ignorance religieuse, l'émigration aux Etats-Unis, les retours au village de ces émigrés, devaient produire la petite légion des « revirés 》 et des témoins de Jéhovah. Maladies morales dont il ne faudrait néanmoins exagérer l'expansion. En ce chapitre des mœurs, dans l'histoire de Chéticamp, nous invitons à lire tout particulièrement les pages du Père Chiasson, sur les contes, les chansons, voire les superstitions des Chéticantins. Là plus qu'ailleurs peut-être l'on goûtera la savoureuse originalité de ces gens de l'autre bout du pays. L'auteur a voulu joindre à ce tableau quelques pages sur le vocabulaire 
de ces Acadiens du Cap-Breton: liste de mots de veine populaire, qui fera la joie des linguistes.

Quel est l'avenir de Chéticamp ? Un mal l'a longtemps rongé: l'émigration vers les régions minières de la NouvelleEcosse, vers les Iles-de-la-Madeleine, l'Ile-du-Prince-Edouard, vers Montréal, vers Toronto, vers les Etats-Unis. Depuis une trentaine d'années, l'établissement des coopératives, des pensions de vieillesse, des allocations familiales ont ralenti cet éparpillement. Chéticamp est maintenant une paroisse de 3,000 âmes. Elle possède des écoles qui, tout en sauvant la foi et la langue française, conduisent à de plus grandes écoles où se sont formées quelques illustrations chéticantines. Pour stabiliser sa population, il faudrait, à Chéticamp, nous dit-on, développer la culture maraîchère, l'élevage, l'industrie laitière, l'aviculture, faire surgir quelques manufactures. Problèmes ardus, mais non point au-dessus de l'énergie d'un petit peuple qui en a résolu tant d'autres. Et c'est bien là la conviction du Père Chiasson qui nous décrit son pays et les gens de son pays avec une sympathie si agréablement contagieuse.

LIONEL GROULX, ptre 